\title{
Carotid Stenting without Angioplasty and without Protection
}

\section{The Advantages of a Less Invasive Procedure}

\author{
M. LEONARDI, M. DALL'OLIO, L. RAFFI, P. CENNI, L. SIMONETTI, \\ R. MARASCO, F. GIAGNORIO* \\ Neuroradiology Department, *Medical Department, Bellaria Hospital; Bologna, Italy
}

Key words: primary carotid stenting, atherosclerotic disease, mini-invasive technique, endovascular, protection device

\section{Summary}

Endovascular stenting is a consolidated alternative to thrombendarterectomy in the treatment of extracranial carotid artery atheromasic stenosis.

The most common complication of stenting is a distal embolism causing clinically silent or symptomatic cerebral ischaemia. To prevent this complication distal embolism protection devices are often used but their effectiveness remains unsettled. In addition, there is some evidence that distal embolism may actually be triggered by the protection systems due to clot formation at their distal surface or in the intimal lesions these systems cause. Another rarer complication is hyperperfusion syndrome arising during both stenting and thrombendarterectomy but more common in endovascular procedures. To avoid these complications the Neuroradiology Service at Bellaria Hospital (Bologna Local Health Trust) has devised a mini-invasive carotid stenting technique that does not require either distal embolism protection or angioplasty.

The technique uses only the radial force exerted by the self-expanding stent to widen the atherosclerotic stenosis slowly and gradually. The goal of treatment has also changed from a prompt restoration of the atheromasic vessel's original calibre to slow transformation of the hemodynamic significance of the stenosis. The technique's success lies mainly in selecting the stenosis to treat using CT angiography to ana- lyse plaque morphology and structure. We used the technique to treat 83 stenotic lesions in 75 patients. The study aims to describe and discuss our experience.

\section{Introduction}

Endovascular stenting a well-established less invasive alternative to thrombendarterectomy (TEA) in the treatment of extracranial carotid artery atheromasic stenosis, namely in symptomatic high-risk surgical candidates ${ }^{1-5}$. Stenting is also a safer primary treatment than angioplasty as it has a lower incidence of adverse events like plaque fragmentation, arterial dissection and acute carotid artery occlusion. Stenting will also establish a more definitive vessel lumen thereby lowering the incidence of subsequent restenosis due to neointimal hyperplasia typical of angioplasty alone ${ }^{6,7}$.

Most operators currently combine carotid stenting with angioplasty either before or after dilating the site of stenosis or only after dilatation.

The advent of technically advanced dedicated carotid artery stents has made it easier for operators to manoeuvre and position stents, shortening procedure times without relinquishing control. In addition, self-expanding carotid stents have significant radial force. Despite these advances, transient or permanent neurologic events responsible for major or minor ischaemia due to embolism or hemodynamic fac- 

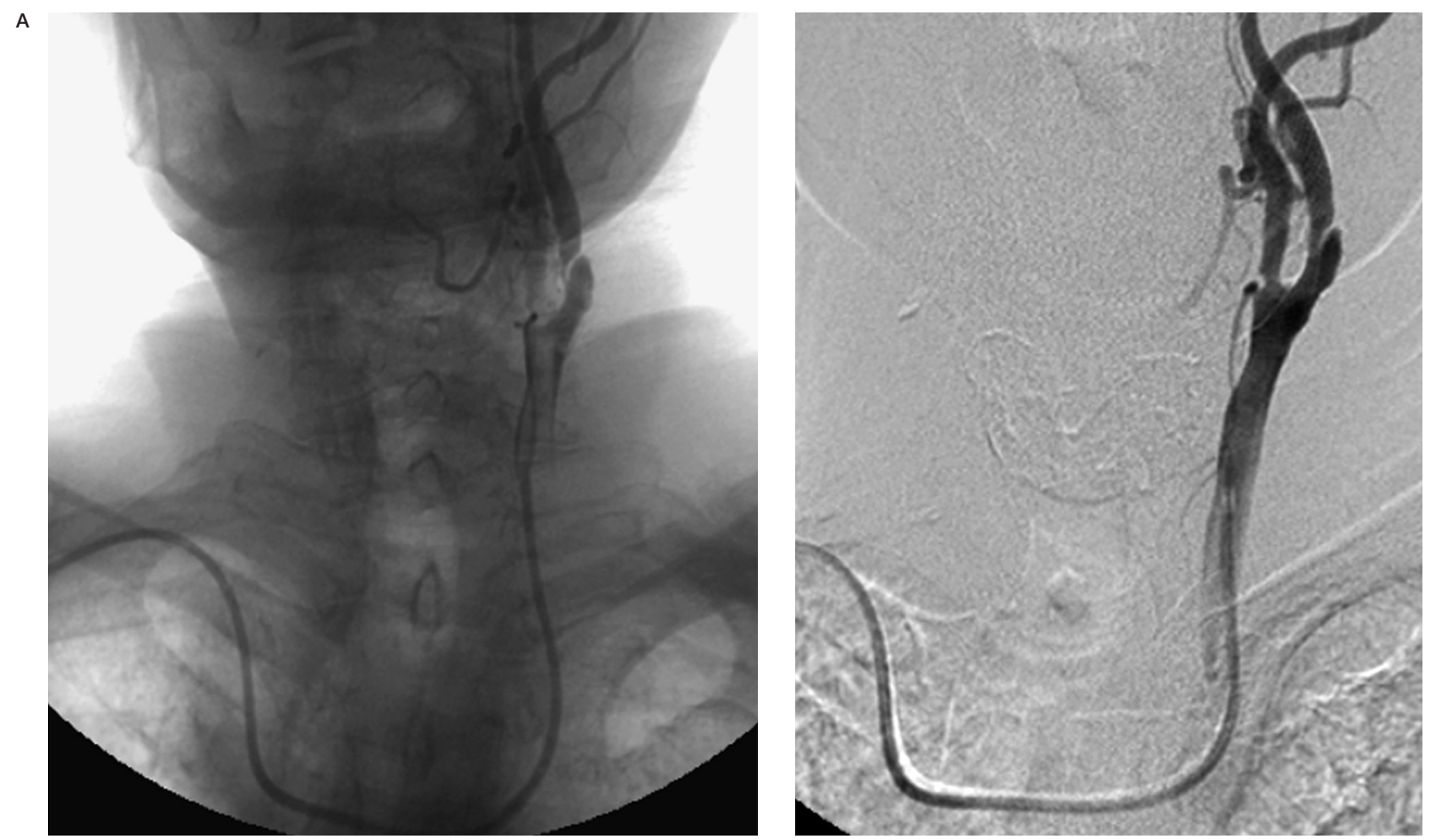

Figure 1 Unsubtracted AP image (A), subtracted AP image (B): the left common carotid artery originating from the arch at an acute angle is easily reached by a radial approach. Note the dissected atherosclerotic plaque at the origin of the internal carotid artery with stenosis of the residual vessel lumen and a small atherosclerotic plaque at the origin of the external carotid artery.

tors (hypo or hyperperfusion) still represent a therapeutic challenge. Most of these events involve embolic infarcts (micro or macroemboli) generated in the most hazardous stages of the endovascular procedure, i.e. the passage of the guidewire through the stenosis, predilation, stent positioning and postdilation. Unfortunately current literature reports fail to distinguish the complications caused by stenting from those due to angioplasty. To have a rough evaluation we must go back to the time of angioplasty alone before the advent of stenting when the complication rate of angioplasty without filter was around $10 \%$. Nowadays the complication rate of angioplasty with stenting and filtering is around 3,3\%.

To prevent complications various protection systems are used, most commonly the filter protection device. However, the success of filter protection is linked to factors intrinsic to the filter itself (its profile, capacity to control the system, diameter of the open filter and its length and pore size) and extrinsic factors (ability of the filter to work in tortuous carotid axes, likelihood of carotid spasm) and lastly to factors linked to filter withdrawal. In addition, there have been some reports of filter-induced intimal and subintimal histological damage influencing the number of intracerebral embolic events ${ }^{8-14}$.

This paper describes a cohort of patients treated by carotid stenting at the Neuroradiological Service at our hospital. Most of our patients were treated by a mini-invasive carotid stenting procedure we devised which does not require either prior/post dilation or mechanical protection devices. In our opinion this approach avoids angioplasty-related complications.

\section{Material and Methods}

We retrospectively reviewed all carotid stenting procedures undertaken between January 2000 and December 2007 at the Neuroradiology Service of Bellaria Hospital, Bologna. The diagnosis of carotid artery stenosis had been established in all patients by echo Doppler examination and confirmed by CT angiography using multislice systems (Philips, GE and Siemens) to study the state of the arterial wall, plaque morphology and the vascular anatomy of the supraortic arteries to determine the endovascular approach. 
Patients were selected following these major criteria: stenosis exceeding $70 \%$ or unstable symptomatic plaque, calcification of the plaque limited to part of the lesion ${ }^{53}$. Circumferential plaque not allowing stent dilatation is considered, in our series, an indication for a surgical approach. CT is the best imaging system to obtain this information. Examination yielded an accurate in vivo anatomopathological reconstruction of the stenotic site, subsequently confirmed prior to treatment in all cases by biplanar angiography using a GE device. CT angiography disclosed sites of ulcerated plaque in 12 patients, subsequently confirmed by angiography.

We treated 75 patients (53 men; 22 women), average age 69.5 years (range 53-89), positioning 83 stents. Our case series is an intention to treat selected patients.

The endovascular treatment was done after restenosis following surgical thrombarterectomy in nine patients, whereas the remainder had primary carotid stenting. All the remaining stenoses were hemodynamically significant and around half of the lesions were subocclusive. Seven patients (6 men, 1 woman) were treated bilaterally. Two stents were placed on the same side in one patient: one in the common carotid artery, the other in the internal carotid artery.

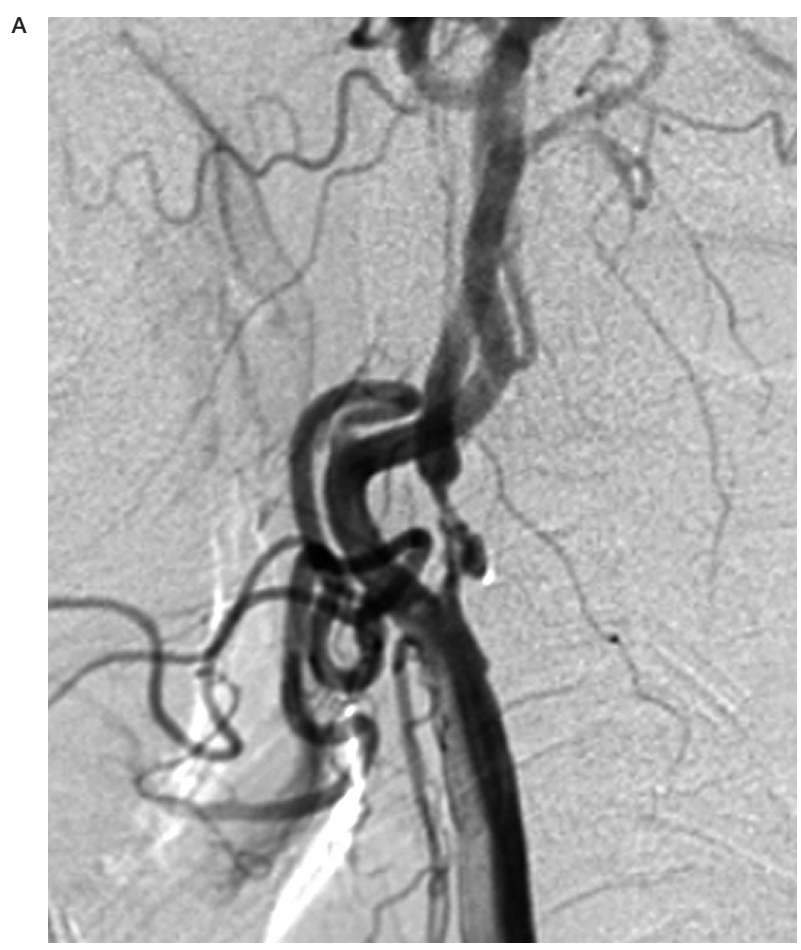

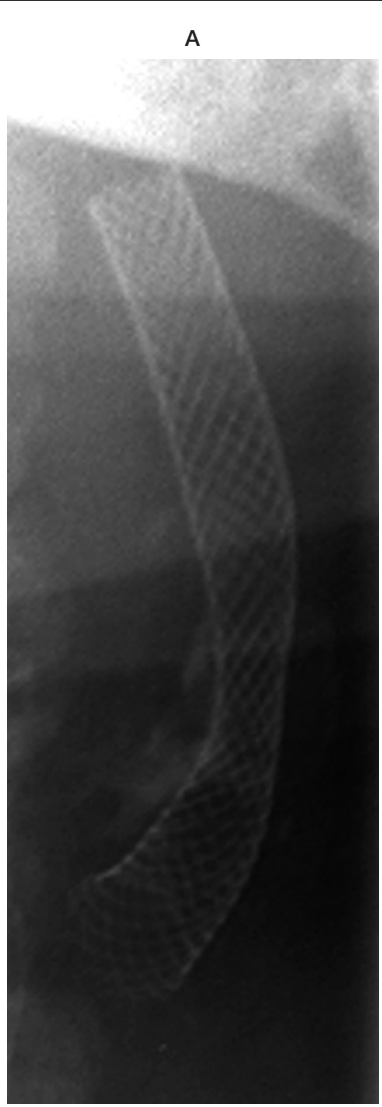

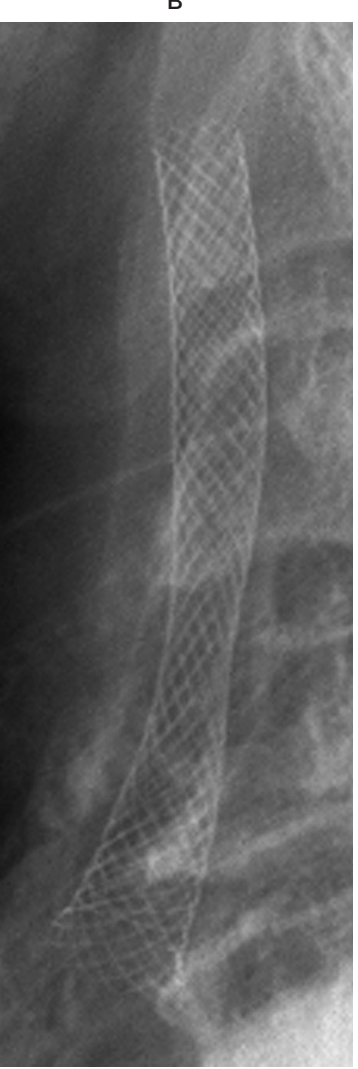

Figure 2 Same patient, Unsubtracted AP image (A), unsubtracted LL image (B): stent dilation just after stent placement with widening of the stenosis.

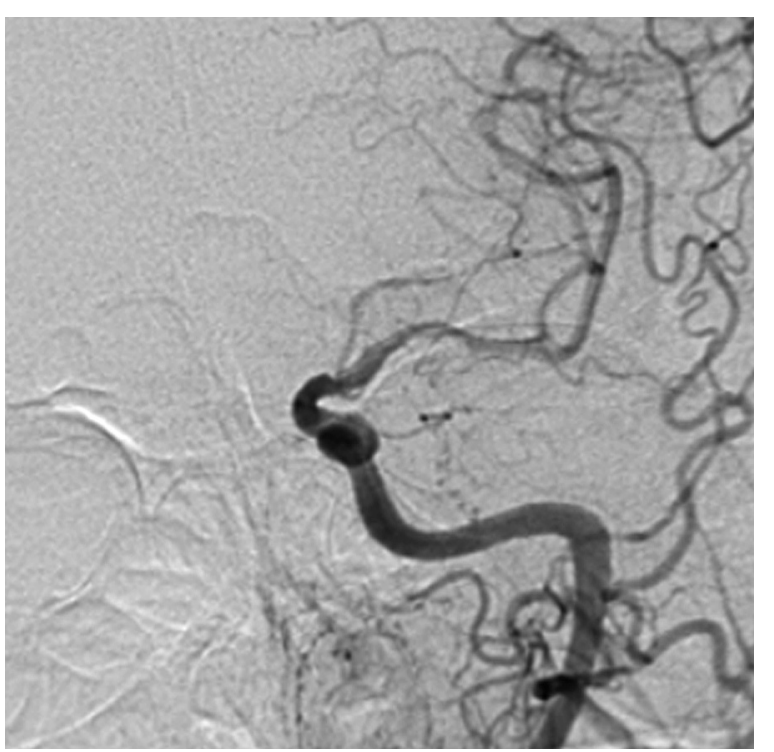

Figure 3 A) Lateral view showing ulcerated atherosclerotic plaque at the origin of the left internal carotid artery causing severe stenosis. B) Intracranial AP view showing reduced flow in the middle cerebral artery and especially in the non opacified anterior cerebral artery. 


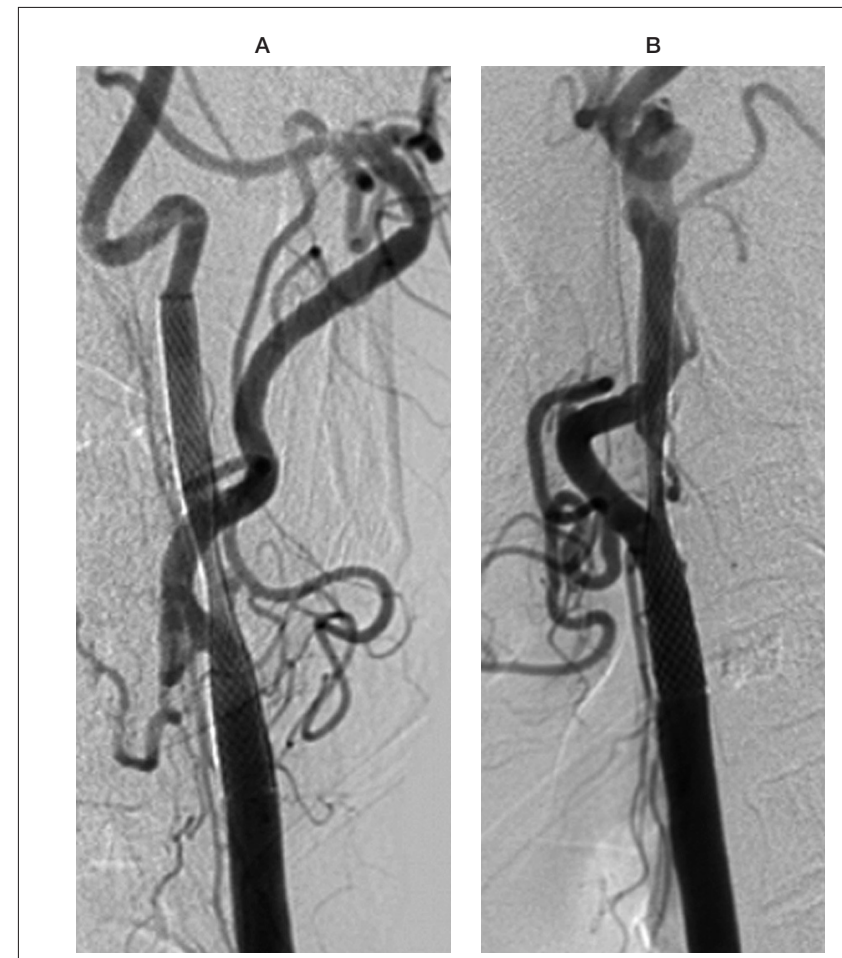

Figure 4 Same patient. A) AP view, (B) lateral view: stent placemnent with widening of the stenosis and blood trapped within the ulceration.

Two stents were positioned during the same interventional session in four patients, and in four separate sessions in the remainer. All the remaining patients underwent monolateral stenting. A single stent was positioned astride a double consequential carotid stenosis (tandem le-

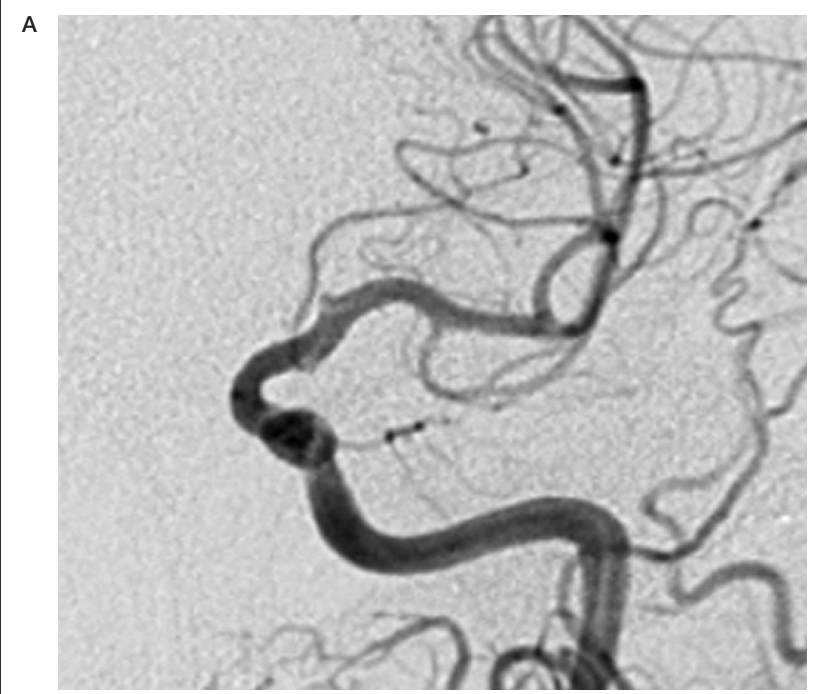

sions) in two patients. Six patients had intracranial aneurysms (tandem lesions), four of which were embolized during the same interventional session. Three patients were treated using a radial approach (figure 1,2).

Most procedures were carried out under local anaesthesia in the presence of an anaesthesist. The four patients who underwent embolization of an intracranial aneurysm received general anaesthesia.

Medical management after the endovascular procedure differed according to the treatment underway before stenting. Most patients receiving anti-aggregant therapy were given a bolus of heparin (5000 units) during stenting followed by enoxeparin (4000 units) for two days after the procedure, ticlopidine (250 $\mathrm{mg} \times 2$ ), acetylsalicylic acid ( $0.3 \mathrm{~g} \mathrm{x} 1)$ and omeprazole for one month post-treatment, and only aspirin (250 g x 1) for the following six months. Patients receiving oral anticoagulants (warfarin) were not given the heparin bolus during the procedure but only aspirin $1 \mathrm{~g}$ i.v. The previous anticoagulant therapy was resumed after stenting in addition to acetylsalicylic acid (0.3 $\mathrm{g} \mathrm{x} 1)$ and omeprazole for six months.

Two carotid access techniques were mainly used. The first required an $8 \mathrm{~F}$ guiding catheter (Cordis Neurovascular FL, Boston Scientific CA) with a straight or slightly curved tip depending on the vascular anatomy, positioned in the common carotid artery through which the stent was inserted.

Figure 5 Same patient. Early (A) and later (B) angiographic sequences showing an immediate increase in perfusion in both the middle and anterior (opacified) cerebral arteries. 


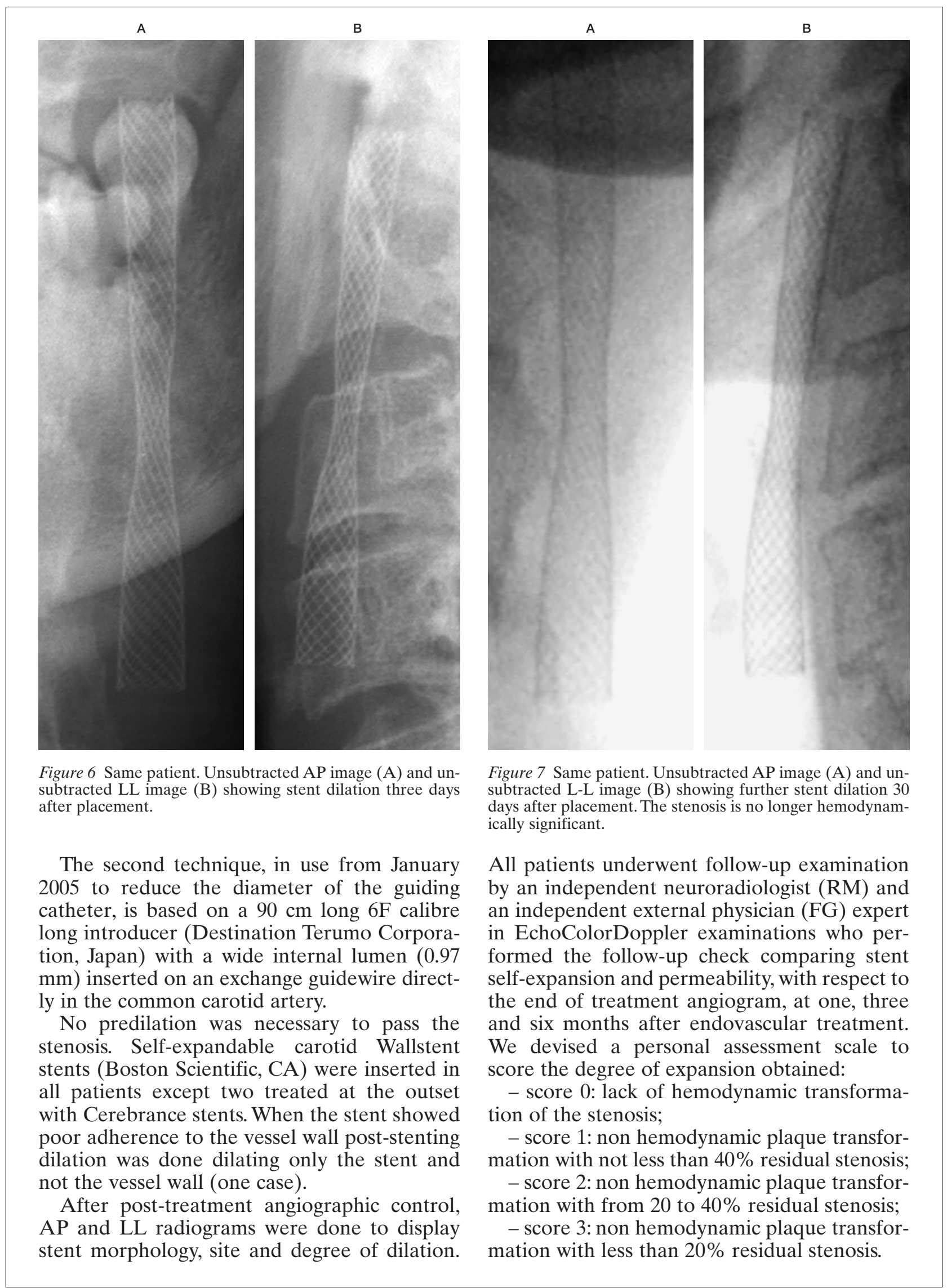



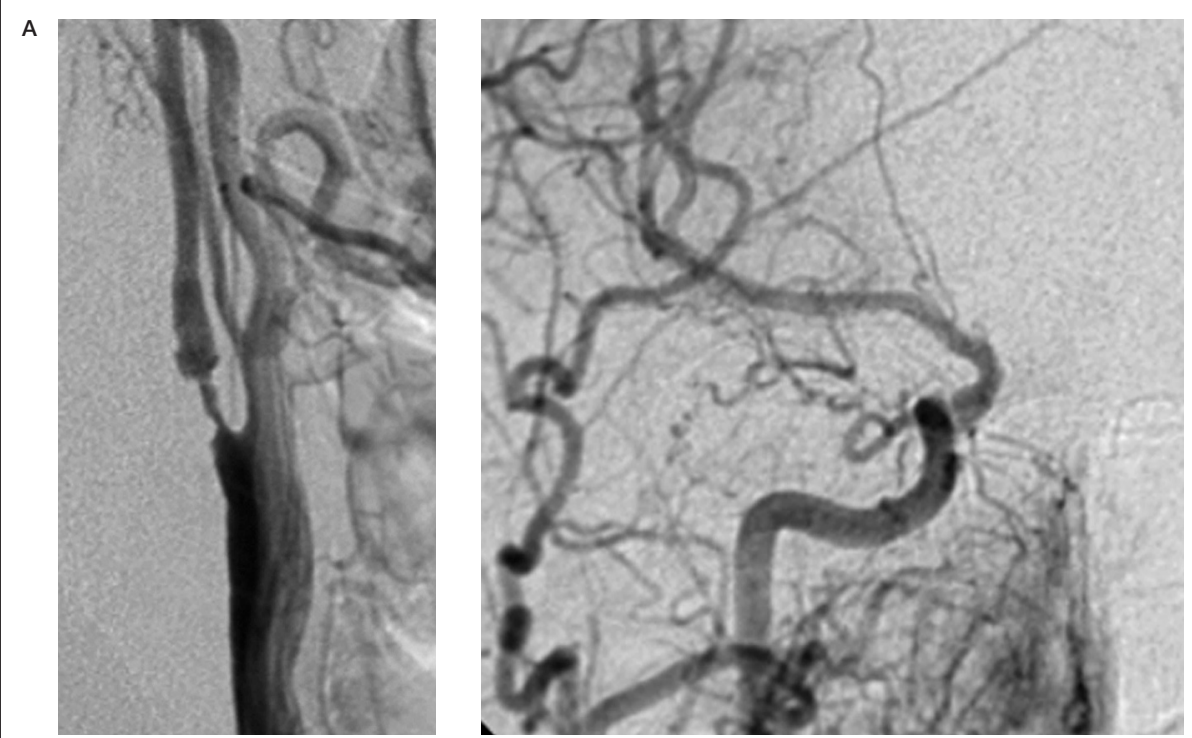

Figure 8 A) Lateral view showing severe stenosis at the origin of the right internal carotid artery. (B) Intracranial AP view: the anterior cerebral artery is not opacified.

\section{Results}

Only one technical failure was encountered among the 83 endovascular procedures. This patient presented a large ulcerated plaque at the left carotid bifurcation and a subocclusive stenosis about $2 \mathrm{~cm}$ from the origin of the right internal carotid artery with signs of reduced right cerebral blood flow with compensation from the circle of Willis, the vertebral and left carotid arteries. A Stent was successfully deployed on the left and controlateral stenting was attempted but failed as a vessel dissection was seen with its definitive occlusion. No deficits were observed on clinical neurological follow-up $24 \mathrm{~h}$ after the procedure. No major or minor ischaemic events or hyperperfusion syndromes were encountered.

Long-term clinical follow-up disclosed two patients who had had transient ischaemic events controlateral to the site of carotid stent deployment, four patients had died from cancer and one patient from acute myocardial infarction. Radiological follow-up was done in 70 patients as 13 failed to present at follow-up visits. The x-ray and EchoColorDoppler assessment yielded the following score: Score 0 (no results): three patients; two asymptomatic stent occlusion (figures 8-10); one post-stenting restenosis treated by angioplasty with cutting balloon (figures 11-13). Score 1: non hemodynamic plaque transformation with not less than $40 \%$ residual stenosis: 25 patients. Score 2: non hemodynamic plaque transformation with from
20 to $40 \%$ residual stenosis: 30 patients. Score 3: non hemodynamic plaque transformation with less than $20 \%$ residual stenosis: 12 patients.

\section{Discussion}

Carotid stenting has become an alternative to thrombendarterectomy (TEA) in the treatment of carotid atheromasic disease ${ }^{15-17}$. In addition to post-TEA restenosis, post-attinic stenosis and primary stenosis in high risk surgical candidates, the good results obtained with carotid stenting have broadened the indications for endovascular treatment to patients with primary stenosis.

The main criticism levelled against stenting as opposed to TEA is the higher risk of distal embolism from plaque detachment after widening the passageway. To avoid this complication various distal embolism protection devices types have been devised:

Balloons to stop blood flow in the internal carotid artery;

Filters allowing blood flow through the filter pores, withholding any fragments of plaque;

Parodi-type inversion of normal blood flow in the internal carotid artery.

Most operators currently use a distal protection device even though their effectiveness remains unsettled. Legal considerations play an important role in opting to use a protection system. Some literature reports claim the benefits of protection devices whereas others are criti- 
$\mathrm{cal}^{18-32}$. Much of this research consists of an analysis of the presence and quantity of material filtered by the protection system after the procedure, or monitoring the cerebral microembolism during endovascular treatment using multifrequency transcranial Doppler which can distinguish solid from gas emboli. Other studies have used MR DWI before and after endovascular treatment to disclose any cerebral ischaemic events, including silent lesions ${ }^{33}$. So-called silent ischaemic events seem to determine a progressive impairment of $\operatorname{cog}$ nitive functions in elderly patients leading to dementia in the severest cases ${ }^{34,35}$. Protection systems do not seem able to withhold all plaque fragments in cases of massive embolism resulting in multiple distal ischaemia. In addition new ischaemic cerebral lesions have been disclosed even when no material had been filtered by the protection device ${ }^{36}$.

This evidence has given rise to a number of pathogenetic theories such as the formation of emboli in distal surface of the protection device and in intimal lesions due to arterial wall contact with the balloon or filter protection device. Whether they favour the use of protection devices or not, most operators agreed on the need to combine carotid stenting with angioplasty ei-
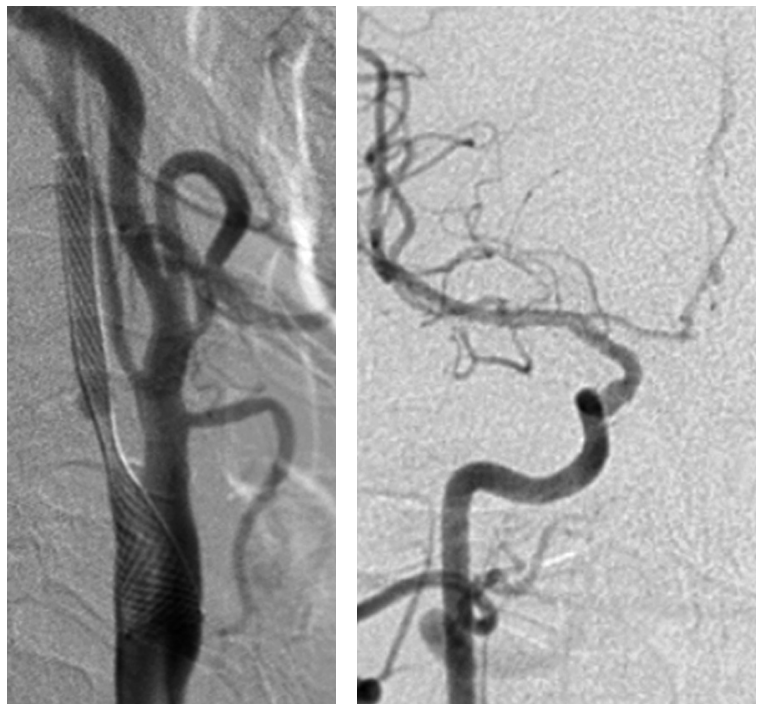

Figure 9 A Lateral view: stent placement fails to widen the stenosis. B) Intracranial AP view showing intracranial perfusion with opacification of the anterior cerebral artery.

ther as a pre and post-dilation procedure or only post-dilation. Angioplasty represents the most traumatic stage of the endovascular procedure when the atherosclerotic plaque is disrupted with rapid restoration of vessel calibre. Plaque rupture can free fragments of plaque

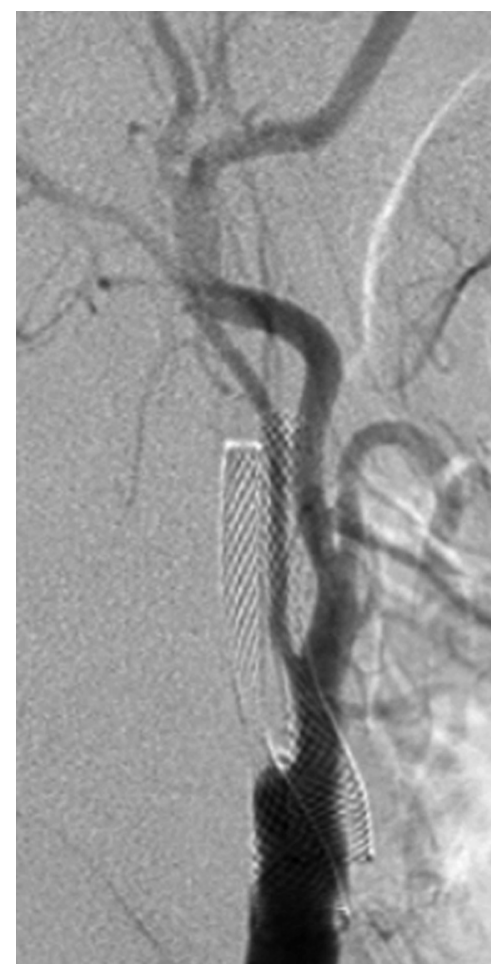

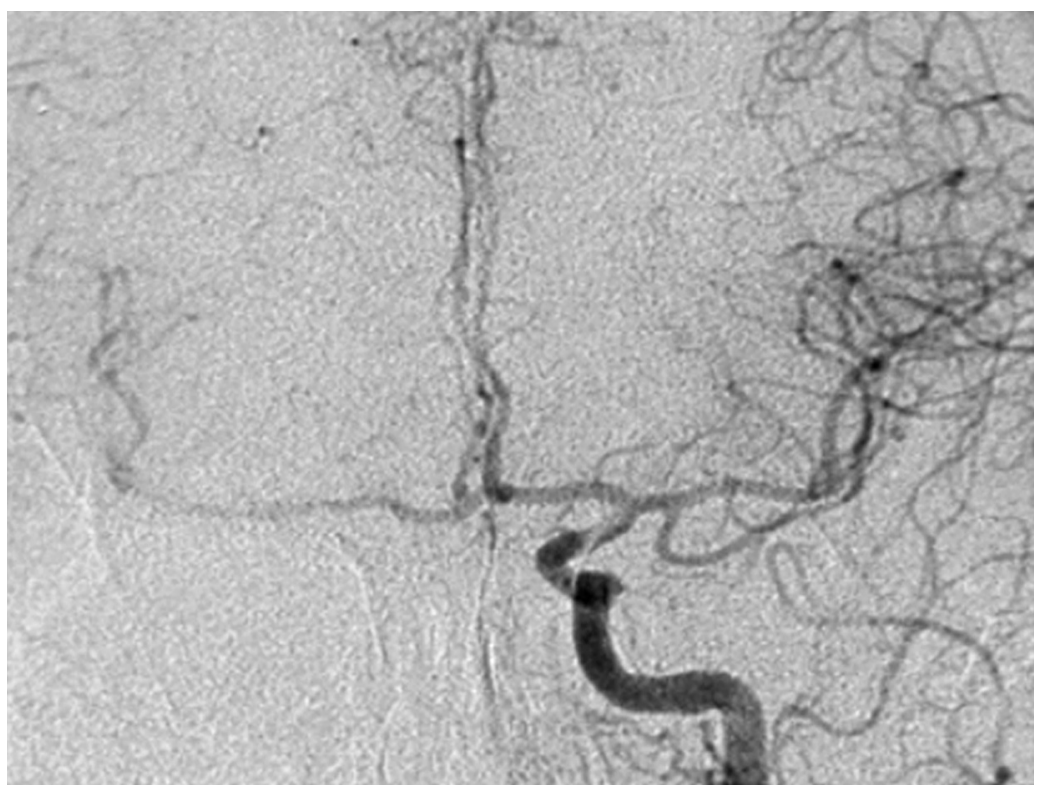

Figure 10 A) Lateral view: nine month follow-up showing an undilated occluded stent. B) Intracrnial AP view: fortunately the occlusion is asymptomatic thanks to compensatory circulation. 


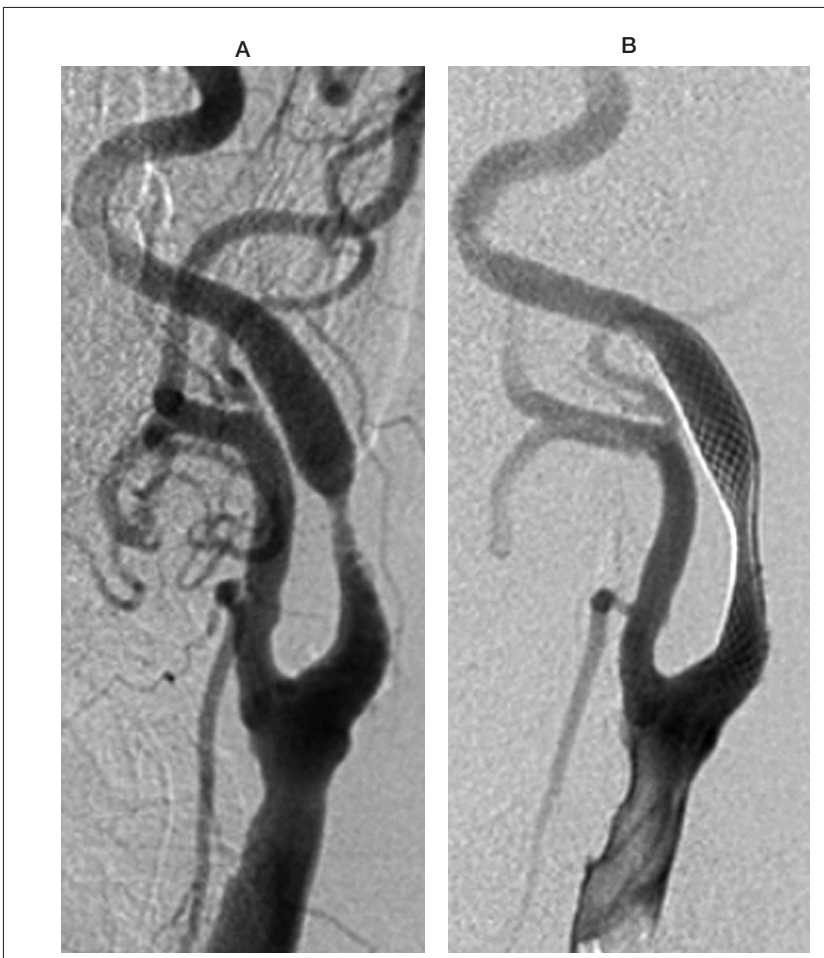

Figure 11 Lateral view (A) showing atherosclerotic stenosis of the internal carotid artery. B) Stent placement.

even after stent deployment despite its containment effect. In addition, angioplasty can lead to bradycardia and hypotension both during and after the procedure requiring atropine administration before dilation.

The prompt restoration of normal vessel calibre produced by angioplasty and TEA can be complicated by hyperperfusion syndrome. This complication may arise during both stenting and TEA but is more common in endovascular procedures $^{37-45}$. One explanation for this may be the greater anticoagulant and antiaggregant situation present during stenting to avoid any complications of angioplasty and protection devices. Hyperperfusion injury can arise with or without cerebral hemorrhage after a delay of five to seven days ${ }^{46}$, or occur several hours after surgery ${ }^{47}$ or endovascular treatment. The severest forms are associated with bleeding, especially early hemorrhage, which may be fatal. Another pathogenetically different type of cerebral hemorrhage may also occur after restoration of normal vessel calibre. This bleed does not arise in the hemodynamically impaired territory, as would be expected in hyperperfusion syndrome, but in the basal nuclei. It may be caused by rupture of the perforating arteries in the basal ganglia following a sudden normaliza- tion of perfusion pressure thus patients with hypertensive microangiopathy at greater risk ${ }^{48}$.

We have devised a primary stenting technique different from the classic procedures or techniques modified to incorporate protection devices ${ }^{49-52}$. Our approach does not require either angioplasty or distal embolism protection. The goal of treatment has also changed from prompt restoration of normal carotid calibre to slow transformation of the hemodynamic significance of the stenosis. Stenting will stabilize the hemodynamic situation over time. We found that the radial force exerted by deployment of a self-expanding Wallstent (Boston Scientific) in selected patients with severe stenosis would progressively widen the stenosis, remodelling the vessel lumen. The presence of ulcerated plaque did not affect the treatment as blood is trapped within the ulceration and thrombosis occurs after the stent is positioned (figure 3-7). A hemodynamically significant positive outcome was obtained in most of our patients in the three days after stent deployment with further widening of the stent at one and three month follow-up and in rare cases after six months. Our stenting technique is based on the following considerations:

The procedure is quick and relatively straightforward. Once the guiding catheter or introducer has been positioned $90 \mathrm{~cm}$ into the common carotid artery, stent deployment lasts from five to ten minutes. The progressive widening of the atherosclerotic plaque due to self-expansion of the stent limits damage to the plaques thereby reducing the risk of embolism and obviating the need for protection devices. Cerebral blood flow is increased and perfusion pressure normalized slowly thereby gradually reducing the possibility of either hyperperfusion syndrome or hemorrhage in the basal nuclei.

The rationale of the procedure is supported by the lack of major or minor ischaemic events, hyperfusion syndrome or hemorrhage in the basal ganglia never observed in our series.

The success of this endovascular approach lies mainly in selecting the stenosis to treat. CT angiography (CTA) is used to analyse plaque morphology, size and structure and the degree of carotid artery stenosis and confirmed by echo colour Doppler findings. CTA analyses the composition of the atheromasic plaque disclosing the prevalence of soft and calcified tissue, and measures the morphology and extension of the atherosclerotic lesion and the resid- 


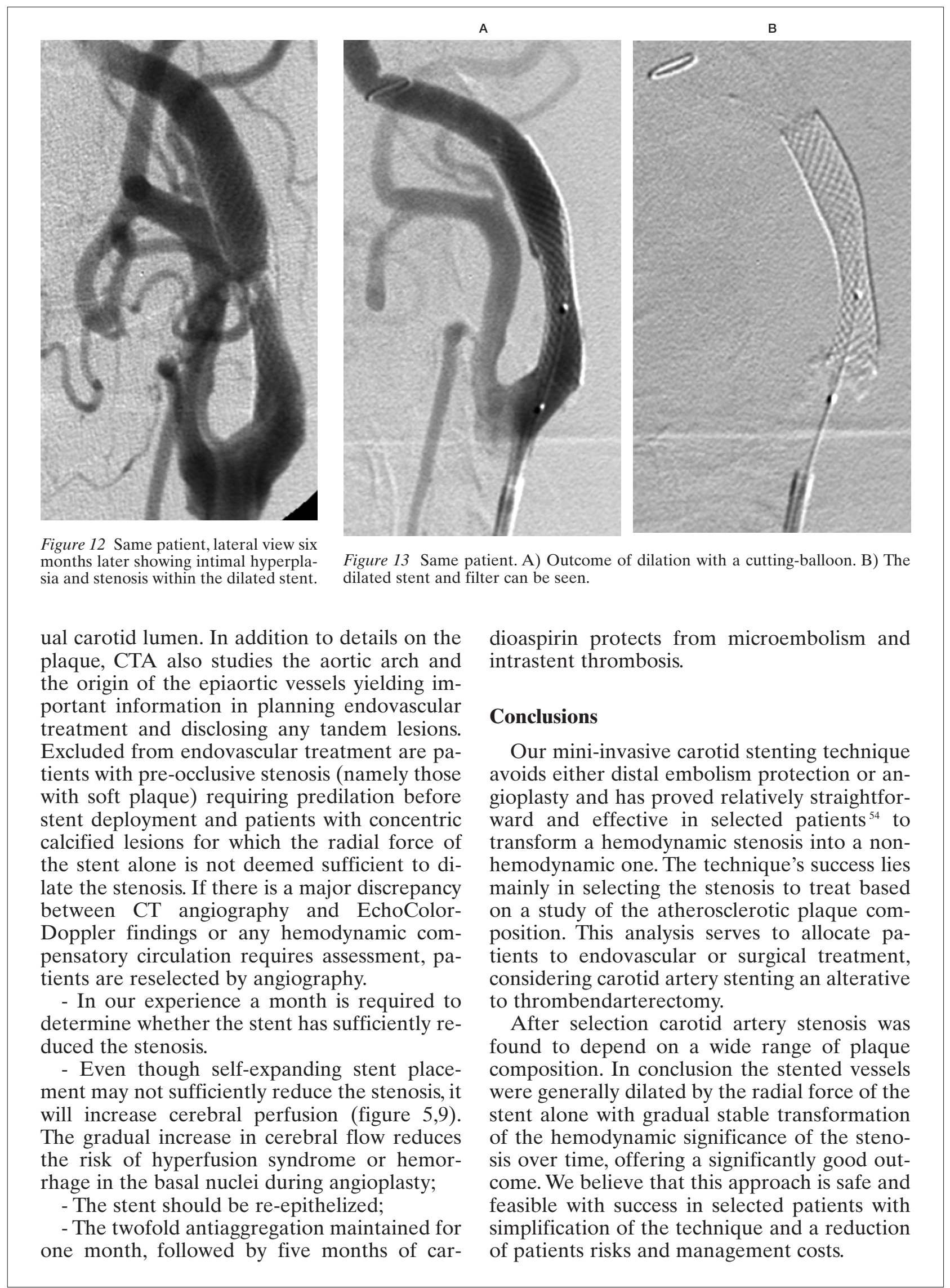




\section{References}

1 Derubertis BG, Chaer RA et Al: Evolution of the treatment of carotid occlusive disease: indications for carotid angioplasty and stenting versus carotid endoarterectomy. Journal Cardiovascular Surgery 47: 297-303, 2006.

2 CAVATAS Investigators: Endovascular versus surgical treatment in patients with carotid stenosis in the Carotid and Vertebral Artery Transluminal Angioplasty Study (CAVATAS): a randomized trial. Lancet 357 1729-1737, 2001.

3 Naylor AR, Rothwell PM, Bell PRF: Overview of the principal results and secondary analyses from the European and the North American randomised trials of carotid endoarterectomy. European Journal Endovascular Surgery 26: 115-119, 2003.

4 Albert MJ: WALLSTENT. Results of a multicenter prospective randomized trisl of carotid artery stenting versus carotid endoarterectomy. Stroke 32: 325, 2001.

5 Ouriel K, Yadav J et Al: The SAPPHIRE randomised trial of carotid stenting versus endarterectomy: a subgroup analysis. Proceedings of the Annual Meeting of the Society for Vascular Surgery and the American Association of Vascular Surgeons. Chicago, $8^{\text {th }}-11^{\text {th }}$ June 2003.

6 Dietrich EB,Ndiaye M, Reid DB: Stenting in the carotid artery: initial experience in 110 patients. J Endovascular Surgery 3: 42-62, 1996

7 Yadav J, Roubin GS et Al: Elective stenting of the extracranial carotid arteries. Circulation 95: 376-381, 1997.

8 Muller-Hulsbeck S, Solzmann P et Al: Vessel wall damage caused by cerebral protection devices: ex vivo evaluation in porcine carotid arteries. Radiology 235: 454460, 2005.

9 Muller-Hulsbeck S, Jahnke T et Al: Comparison of various cerebral protection devices used for carotid stenting: an in vitro experiments. Journal of Vascular Interventional Radiology 14: 613-620, 2003.

10 Muller-Hulsbeck S, Jahnke T et Al: In vitro comparison of four cerebral protection filters for preventing placque embolization during carotid interventions. Journal of Endovascular Therapy 9: 793-802, 2002.

11 Ohki T, Roubin GS et Al: Efficacy of a filter device in the prevention of embolic events during carotid angioplasty and stenting: an ex vivo analysis. Journal of Vascular Surgery 30: 1034-1044, 1999.

12 Grego F, Frigatti P et Al: Prospective comparative study of two cerebral protection devices in carotid angioplasty and stenting. Journal of Cardiovascular Surgery (Torino) 43: 391-397, 2002.

13 Jaeger HJ, Mathias KD et Al: Cerebral ischemia detected with diffusion-weighted imaging after stent implantation in the carotid artery. Am J Neuroradiol 23 200-207, 2002.

14 Rosenkranz M, Fiehler J et Al: The amount of solid cerebral microemboli during carotid stenting does not relate to the frequency of silent ischemic lesions. Am J Neuroradiol 27: 157-161, 2006.

15 Shawl F, Kadro W et Al: Safety and efficacy of elective carotid artery stenting in high-risk patients. J Am Coll Cardiol 35: 1721-1728, 2000

16 Roubin GS, New G et Al: Immediate and late clinical outcomes of carotid artery stenting in patients with symptomatic and asymptomatic carotid artery stenosis: a 5-year prospective analysis. Circulation 103: 532-537, 2001

17 Wholey MH, Wholey $\mathrm{M}$ et Al: Global experience in cervical carotid artery stent placement. Catheter cardiovasc Interv 50: 160-167, 2000.
18 Mas JL, Chatellier G, Beyssen B: Carotid angioplasty and stenting with and without cerebral protection: clinical alert from the endarterectomy versus angioplasty in patients with symptomatic severe carotid stenosis (EVA-3S) trial. Stroke 35: e18-e21, 2004.

19 Kastrup A, Groschel K, Krapf H: Early outcome of carotid angioplasty and stenting with and without cerebral protection devices; a systematic review of the literature. Stroke 34: 813-819, 2003

20 Yadav JS, Wholey MH et Al: Protected carotid-artery stenting versus endarterectomy in high-risk patients. $\mathrm{N}$ Engl J Med 351: 1493-1501, 2004.

21 Cremonesi A, Manetti R et Al: Protected carotid stenting: clinical advantages and complications of embolic protection devices in 442 consecutive patients. Stroke 34: 1936-1943, 2003

22 Bush RL, Kougias P et Al: A comparison of carotid artery stenting with neuroprotection versus carotid endarterectomy under local anesthesia. Am J Surg 190: 696-700, 2005.

23 Kasirjan K, Schneider PA, Kent KC: Filter devices for cerebral protection during carotid angioplasty and stenting. J Endovasc Ther 10: 1039-1045, 2003.

24 Macdonald S, Mckevitt F et Al: Neurological outcomes after carotid stenting protected with the NeuroShield filter compared to unprotected stenting. J Endovasc Ther 9: 777-785, 2002.

25 Lee YH, Kim TK et Al: Simultaneous bilteral carotid stenting under the circumstance of neuroprotection device. Interventional Neuroradiology 12: 141-147, 2006.

26 Jaeger H, Mathias K et Al: Clinical results of cerebral protection with a filter device during stent implantation of the carotid artery. Cardiovasc Intervent Radiol 24: 249-256, 2001

27 Schluter M, Tubler T et Al: Focal ischemia of the brain after neuroprotected carotid artery stenting. J Am Coll Cardiol 42: 1007-1013, 2003.

28 Jaeger H, Mathias K et Al: Diffusion-weighted MR imaging after angioplasty or angioplasty plus stenting of arteries supplying the brain. Am J Neuroradiol 22:1251-1259, 2001.

29 Flach HZ, Ouhlous M et Al: Cerebral ischemia after carotid intervention. J Endovasc Ther 11:251-257, 2004.

30 Bonaldi G, Minonzio G et Al: Brain protection with intracarotid filter in angioplasty and stenting of the cervical carotid bifurcation. Preliminary experience in five cases. Interventional Neuroradiology 7: 103-110, 2001.

31 Bonaldi G: Angioplasty and stenting of the cervical carotid bifurcation: report of a 4-year series. Neuroradiology 44: 164-174, 2002.

32 Bonaldi G, Aiazzi L et Al: Angioplasty and stenting of the cervical carotid bifurcation under filter protection: a prospective study in a series of 53 patients. J Neuroradiol 32: 109-117, 2005.

33 Derdeyn CP: Diffusion-weighted imaging as a surrogate marker for stroke as a complication of cerebrovascular procedures and devices. Am J Neuroradiol 22: 1234-1235, 2001

34 Vermeer SE, Prins ND et Al: Silent brain infarcts and the risk of dementia and cognitive decline. New Engl J Med 348: 1215-1222, 2003

35 Fearn SJ, Pole R et Al: Cerebral injury during cardiopulmonary bypass : emboli impair memory. J Thorac Cardiovasc Surg 121: 1150-1160, 2001.

36 Maleux G, Demaerel P et Al: Cerebral ischemia after filter-protected carotid artery stenting is common and cannot be predicted by the presence of substantial amount of debris captured by the filter device. Am J Neuroradiol 27: 1830-1833, 2006. 
37 Abou-Chebl A, Yadav JS et Al. Intracranial hemorrhage and hyperperfusion syndrome following carotid artery stenting: risk factors, prevention, and treatment. J Am Coll Cardiol 43: 1596-1601, 2004.

38 Meyers PM, Higashida RT et Al: Cerebral hyperperfusion sindrome after percutaneous transluminal stenting of the craniocervical arteries. Neurosurgery 47: 335$343,2000$.

39 Ho DS, Wang Y et Al: Epileptic seizures attributed to cerebral hyperperfusion syndrome after percutaneous transluminal angioplasty and stenting of the internal carotid artery. Cerebrovasc Dis 10: 374-379, 2000.

40 McCabe DJ, Brown MM, Clifton A: Fatal cerebral reperfusion hemorrhage after carotid stenting. Stroke 30: 2483-2486, 1999

41 Phatouros CC, Meyers PM et Al: Intracranial hemorrhage and cerebral hyperperfusion syndrome after extracranial carotid angioplasty and stent placement. Am J Neuroradiol 23: 503-504, 2002.

42 Henderson RD, Phan TG et Al. Mechanism of intracranial hemorrhage after carotid endarterectomy. J Neurosurg 95: 964-969, 2001.

43 Reigel MM, Hollier LH et Al: Cerebral hyperperfusion syndrome: a cause of neurologic dysfunction after carotid endarterectomy. J Vasc Surg 5: 628-634, 1987.

44 Schroeder T, Sillesen H et Al: Cerebral hyperperfusion following carotid endarterectomy. J Neurosurg 66: 824$829,1989$.

45 Solomon RA, Luftus CM et Al: Incidence and etiology of intracerebral hemorrhage following carotid endarterectomy. J Neurosurg 64: 29-34, 1986.

46 Sundt TM Jr, Sharbrough FW et Al: Correlation of cerebral blood flow and electroencephalographic changes during carotid endarterectomy: with results of surgery and hemodynamics of cerebral ischemia. Mayo Clin Proc 56: 533-543, 1981.

47 Pomposelli FB, Lamparello PJ et Al: Intracranial hemorrhage after carotid endarterectomy. J Vasc Surg 7: 248-255, 1988.

48 Buhk JH, Cepek L, Knauth M: Hyperacute intracerebral hemorrhage complicating carotid stenting should be distinguished from hyperperfusion syndrome. Am J Neuroradiol 27: 1508-1513, 2006.
49 Henry M, Amor M et Al: Endovascular treatment of atherosclerotic internal carotid artery stenosis. J Endovasc Surg 4: 414, 1997

50 Henry M,Amor M: Carotid angioplasty. The technique. In Henry M, Amor M (ed): Ninth international course book of peripheral vascular intervention. Europa Edition, Paris: 553-566, 1998.

51 Mathias KD, Jager H, Gissler M: Carotid angioplasty and stent placement: a 20 -year experience. In: Henry M. Amor M (ed): Ninth international course book of peripheral vascular intervention. Europa Edition, Paris: 591-599, 1998.

$52 \mathrm{Li} \mathrm{SM}, \mathrm{Li} \mathrm{D}$ et Al: Carotid artery stenting: Experience of a single Institute in China. Interventional Neuroradiology 11: 205-212, 2005.

53 North American Symptomatic Carotid Endarterectomy Trial (NASCET) Steering Committee: North American Symptomatic Carotid Endarterectomy Trial: methods, patient characteristics, and progress. Stroke 22: 711-720, 1991

54 Mynar M, Baldi S et Al: Carotid stenting without use of balloon angioplasty and distal protection devices. Preliminary experience in 100 cases. Am J Neuroradiol 28: 1378-1383, 2007.

Prof. M. Leonardi

Neuroradiology Department

Bellaria Hospital

Via Altura, 3

40139 Bologna, Italy

E-mail: marco.leonardi@ausl.bologna.it 